\title{
A Circular Commons for Digital Devices
}

\section{Tools and Services in eReuse.org}

\author{
David Franquesa \\ Universitat Politècnica de \\ Catalunya \\ Barcelona, Spain \\ david.franquesa@ac.upc.edu leandro@ac.upc.edu
}

\author{
Xavier Bustamante \\ Universitat Politècnica de \\ Catalunya \\ Barcelona, Spain \\ xavierb@ac.upc.edu
}

\begin{abstract}
Circular economies are particularly relevant in the context of digital devices or electric and electronic equipment (EEE). Many digital devices built using scarce and potentially toxic materials have a too-short life, instead of being repaired or reused. In addition, informal recycling of electronics in the developed and developing world has emerged as a new global environmental concern. We describe the dimensions of the problem, the challenge to move to a circular economy, and the ecology for digital devices as well as how this depends on the traceability of devices and cooperation among all stakeholders locally and globally. Moreover we examine the need for support mechanisms to facilitate, standardise, and reduce the transaction cost of the processes and increase their added value. We present eReuse.org, a set of open-source tools, procedures, open data, and services organised as a common-pool resource (CPR) to reach the circular economy of electronics through promoting reuse and ensuring traceability until recycling. Further, eReuse.org envisions empowering and engaging people around the world to create local communities that bootstrap electronic reuse and to support the development of a globally recognised reuse quality and traceability standard.
\end{abstract}

\section{CCS Concepts}

-Human-centered computing $\rightarrow$ Social content sharing; •Networks $\rightarrow$ Location based services; •Hardware $\rightarrow$ Impact on the environment; •Social and professional topics $\rightarrow$ Governmental regulations;

\section{Keywords}

Digital devices; circular economy; reuse; WEEE; traceability

\section{INTRODUCTION}

In the world, there are more digital devices, such as desktops, laptops, tablets, and mobiles, than people. Electronic

\footnotetext{
Definitive Version of Record in the ACM Digital Library: https://dl.acm.org/citation.cfm?id=2926684
}

waste (e-waste) is the largest waste stream, most of it discarded in the general waste stream leading to a loss of secondary resources [2]. Additionally, e-waste is often shipped illegally to developing countries [5]. In Europe, only $35 \%$ is officially reported and collected [4]. The rest is handled by informal workers, degrading the environment [19]. Landfills are still the predominant means of disposal, which poses a threat to the environment and to human health [21]. Although the volume of e-waste is growing three times faster than other types, recycling plants in the EU collect less ewaste [3].

The current model of consumption of high technology is clearly unsustainable. Mobile phones require many rare and precious metals in their construction [17], leading to a potential irrecoverable loss of resources and an effect on other categories, such as global warming, human toxicity, and metal depletion [11]. Moving towards a circular economy must go beyond just recycling materials at a product's end-of-life. A circular economy is one that aims to keep products, components, and materials at their highest utility and value at all times. Repair and reuse implies maintaining a product in use, unlike recycling which indicates the death of the product.

These days, many technological devices restrict the way users can repair the device or replace parts if hardware breaks or becomes outdated. For example, special tools are needed to open most smartphones, and replacing a battery is not possible without risking the warranty on many phones, tablets, and computers [20]. Maestri and Wakkary [12] argued that technology should be designed such that everyday users can repair them - a vision that seems far-fetched given that most products have gone the other direction in recent years by restricting their repairability, disregarding durability in product design, and even artificially limiting the useful life of a product (built-in obsolescence). This could and should change entirely in a future of collapse.

The overall potential of reuse of digital devices can be compared to the number of devices renewed annually, in the range of billions, but a large fraction is dismantled well before the end of usable life. This results in most electric and electronic equipment (EEE) being recycled too early, despite the demand for reuse coming particularly from social organisations [10]. According to a survey by Flash Eurobarometer[8], on average, $50 \%$ of people in Europe would be happy to buy second-hand electronic goods.

The reuse of digital devices consists of finding (by resale or donation) a new user who wants to continue using devices that are no longer needed. If products are repaired, they last 
longer, reducing expenditure in new consumer goods, creating jobs, and strengthening digital skills [9]. Furthermore, considering participation in digital society a human right [6], reuse (donation to charities) can also help to reduce the digital divide and strengthen institutions and projects for social change.

However, reuse is still a minority practice due to the lack of structural support. In public institutions, more than $80 \%$ of digital devices to be recycled are still operational (without need for repair) [10]. Unfortunately, most of them, when depreciated or out of guarantee, are recycled instead of fixed, upgraded, or reused. Civil society entities are willing to promote the positive aspects of reuse of digital devices, but there are no structured services large enough to handle digital devices for reuse. Therefore, they do not know where to turn, as there are no circuits that allow the public administration to redistribute these devices without an extra bureaucracy to decide who should prepare for reuse, who should receive the devices, and how to ensure proper recycling after reuse, given that informal recycling of electronics has emerged as a global environmental concern.

This paper describes the potential and challenges for reuse of digital devices and the need for traceability and support tools to facilitate the process, increase reliability, and reduce cost. This is addressed by eReuse.org, organised as a circular ecology commons. The work is supported by the experience of a decade of field work at the UPC university in Barcelona. During the last four years, more than 1,600 reused computers were donated to more than 900 projects in the area of Catalonia. The tools and services described here have been developed in that period, with a community of more than 10 entities making use of this ecosystem, resulting in more than 10,000 computers processed per year.

The remainder of this paper is structured as follows. Section 2 presents the requirements regarding preparation for reuse and traceability of devices to ensure final recycling. Section 3 presents the principles of the governing mechanisms. In Section 4, we discuss how reuse promotes the model of computing as a service instead of a property. Section 5 introduces the software tools and services developed to implement these principles and some of the implications. Finally, conclusions are presented in Section 6.

\section{CIRCULAR ELECTRONICS}

Refurbishment, reuse, and the correct dismantling process of digital devices effectively contribute to generating a circular economy on electronics. Guvendik [11] presented four lifecycle analysis scenarios using the ReCiPe method over Fairphone [13]. The baseline scenario is the three-year use. Scenario 1 is used for two years, and Scenario 2 represents the first step of Fairphone to create a circular system, which is extending the product lifetime to six years with refurbishment activities. The third scenario (circular), in addition to an extended lifetime of six years, is a dismantling process put into practice when the phone reaches the end of its functional lifetime.

If Fairphone customers use their device for only two years (scenario 1), there will be an increase in metal depletion by $21 \%$, climate change by $18 \%$, and human toxicity by $24 \%$ when compared to the baseline (three years). In the refurbishment scenario (Scenario 2), the reductions over baseline will be $35 \%$ in metal depletion, $16 \%$ in climate change, and $26 \%$ in human toxicity. A circular scenario (third) repre-

\begin{tabular}{|l|ccccc|ccc|}
\hline \multirow{2}{*}{ Smartphone } & \multicolumn{5}{|c|}{ Climate Change, GWP(C02-e) } & \multicolumn{3}{c|}{ Specifications } \\
\cline { 2 - 10 } & Production & Transportation & Use & EoL & Total & $\begin{array}{c}\text { Lifetime } \\
\text { (yr) }\end{array}$ & $\begin{array}{c}\text { Weight } \\
\text { (gr) }\end{array}$ & $\begin{array}{c}\text { Screen size } \\
\text { (") }\end{array}$ \\
\hline \hline Fairphone & 5.3 & 2.7 & 7.9 & 0.1 & 16.0 & 3 & 163 & 4.3 \\
Nokia Lumia 820 & 10.9 & 2.2 & 2.7 & 0.2 & 16.0 & 3 & 160 & 4.3 \\
Nokia Asha 311 & 5.7 & 1.7 & 1.5 & 0.1 & 9.0 & 3 & 98 & 3 \\
Nokia Lumia 1520 & 26.6 & 1.9 & 8.1 & 0.4 & 37.0 & 3 & 209 & 6 \\
iPhone 4S & 35.2 & 3.9 & 14.9 & 1.1 & 55.0 & 3 & 140 & 3.5 \\
iPhone 5c & 43.8 & 2.4 & 12.6 & 1.2 & 60.0 & 3 & 132 & 4 \\
iPhone 5S & 62.3 & 3.0 & 9.0 & 0.8 & 75.0 & 3 & 112 & 4 \\
Sony Xperia T & 35.7 & 5.1 & 9.2 & 1.0 & 51.0 & 3 & 169 & 4.55 \\
Sony Ericsson W890 & 11.2 & 2.2 & 3.4 & 0.2 & 17.0 & 3.5 & 78 & 2 \\
\hline
\end{tabular}

Figure 1: Comparison of smartphone specifications and global warming potential $[1,7,14,16,15]$.

sents significant reductions: $53 \%$ in metal depletion, $24 \%$ in climate change, and $42 \%$ in human toxicity.

Under the preliminary taxonomy for sustainable computing by [22], today's effect on the smartphone industry would at Level 3 (adversely affects sustainability and increases the rate of net resource depletion) even in the circular scenario since their use is only growing.

Without a reuse quality standard and guarantee of traceability, it is uncertain whether non-functional EEE will be exported and end up polluting the environment. Traceability consists of tracing the geographical places, reuse agents, and receivers of digital devices until the collection and recycling points. Traceability is the key to ensuring that reuse contributes to recycling and to avoiding an extended lifetime of a device resulting in uncontrolled waste. For now, we cannot guarantee that a reused device is recycled (and does not end up polluting) or where it has been recycled. However, traceability is difficult; software tools are required to keep track of EEE. It is difficult to uniquely identify digital devices and their components across a deviceâẮ́s life, as it can change. It is also difficult to account for reuse and know whether a device is finally counted twice when reused and recycled. Donors fear that, after reuse, their electronic devices may not end up being recycled, and this could severely affect their goals and image if such devices end up polluting the environment. We claim that traceability in EEE requires a universally unique identifier for each component.

The life cycle of electronics, depicted in Figure 2, essentially contains these phases: production, manufacturing, transfer (retail, donation), use, collection, recycling, and preparation for reuse. We have three loops: product, waste, and recycling (Figure 2). Preparation for reuse usually consists of identifying the product and refurbishing it (e.g., ensure correctness, upgrade, repair, and clean). The recycle loop is the traditional loop where products are collected, pre-processed, and end-processed to recover resources and segregate hazardous waste. The product loop aims to keep products and components alive as long as possible, performing basic preparation for reuse consisting of repairing, upgrading, or transferring by reselling or donating, avoiding waste. The waste loop starts when a product reaches a "collection" point, becoming waste, and it can only return to being a product if it is prepared for reuse under a certified process. This loop mainly has agents, such as reuse, recycling centres, and collection points.

Moreover, eReuse.org provides software tools to assist in collecting data about devices and each step across its life cycle to build a global log of device information. It also 


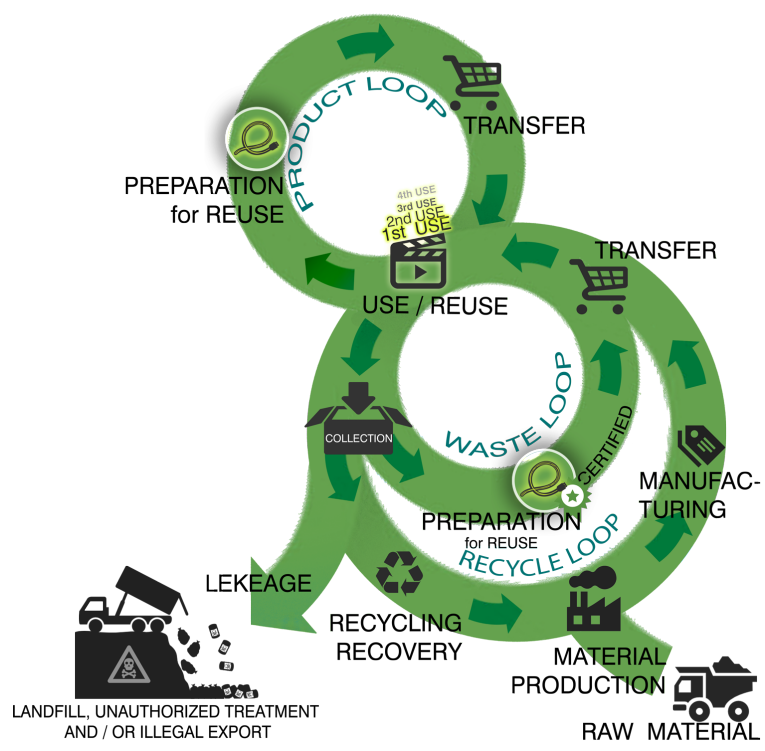

Figure 2: Reuse loops in the life of a digital device.

provides software tools to assist in dealing with each device by 1) collecting information about it; 2) managing inventories of devices; 3 ) preparing a device for reuse by uniquely identifying it, reliably erasing its data, performing tests and benchmarks, and re-installing the OS; 4) finding the next actor in the chain matching the characteristics of the device and its location (can be another end-user or an intermediary organisation preparing or refurbishing the device for the next usage); and finally 5) registering the disposition of a device for recycling.

Our contribution to the product loop is to enable direct transfer of those digital devices to the next agent in the life cycle. Our contribution to the waste loop is to certify the process of preparation for reuse to distinguish an item that is reusable. We provide tools for both cycles (product and waste) to map good-quality used devices and data. In addition, we provide algorithms to determine the reuse with the greatest potential and to allow traceability to ensure final recycling. Moreover, we provide tools for certified data cleaning, communication of the social and environmental value of reuse, and establishment of a system to reward donors and ensure the commitment of the receivers of reused devices to final recycling.

\section{THE EREUSE.ORG COMMONS}

The eReuse.org commons represents a global federation of local groups, organisations, and communities that deal with the circular life of digital devices in their target communities under specific business models. They cooperate to share information, methods, services, and tools under a common governance constituting an ecosystem around circular electronics. For instance, Reutilitza.cat is a community that coordinates direct donation of computers in good condition to charities for free, while Andròmines is an organisation that focuses on collecting a large number of computers from organisations for refurbishment, customisation, and sale for the cost of preparation. Both communities participate and benefit from the eReuse.org commons, and their web portals are federated with the eReuse.org data warehouse and take advantage of common tools and services.

The fundamental principles of eReuse.org, defined at the start to be fully inclusive, revolve around 1) the openness of access (usage and contribution) and 2) the openness of participation (development, operation, and governance) of this resource system. These include a set of software tools, services, and global open traceability data around the life cycle of digital devices and its community.

Non-discriminatory open access. The access to this resource system is non-discriminatory because the maintenance costs are shared among participants in a cost-oriented manner (vs market-oriented), which means free in many cases. It is open because everybody has the right to contribute and access data or use supporting software tools or services to manage digital devices.

Open participation. Everybody has the right to join the community. According to roles and interests, four main groups of stakeholders can be identified.

i) Volunteers, organised in thematic communities (web portals), concerned with aspects such as environmental sustainability, governance of the resource system, software development, repairing, protection of consumers' rights, protection of citizen's rights, and protection of the environment. Volunteers can contribute in identifying and promoting contribution (registering devices into the system), management, and allocation of digital devices to future users according to needs or social support.

ii) Professionals, interested in added value services in order to repair, refurbish, enhance, or recycle second-hand devices, or (re-)manufacturers, interested in producing or selling new components or devices. Professionals and (re-)manufacturers can have their own web portals or participate in one.

iii) Customers, interested in having reused devices or simply cheaper devices.

iv) Public administrations, interested in managing specific attributions and obligations to regulate the participation of society, monitoring environmental effects and data protection, and satisfying their own needs regarding devices.

A balance among these four groups must be preserved, as every group has natural attributions that should not be delegated or undertaken by any other.

Digital devices are rivalrous goods that typically can be used by only one person at the same time and several across each reuse cycle. Although the ownership of a digital device is generally excludable through its acquisition cost, reuse initiatives can reduce that cost dramatically, even to zero economic cost for receivers involved in socially supported activities. In addition, participation in a circular economy of reuse is non-excludable as a goal through many forms of reuse after a significant event (e.g., donation, sale, repair, upgrade, and refurbishment). Therefore, under a reuse framework, digital devices can qualify as common goods (non-excludable and rivalrous).

These fundamental principles, applied to a circular economy of digital devices, result in a resource system that is a collective good, socially produced, and governed as a commonpool resource (CPR) [18]. 
The tools, services, and data are collective goods or peer property in which participants contribute their efforts and goods (data, tools, services, procedures, and certificates) that are shared among all stakeholders to build the resource system, which results in a peer property, provided that the community rules, as a community licence, are respected by all participants.

The development of this economy is a social production, also called peer production, because the participants work cooperatively at the local scale to deploy the tools and services to build local organised islands and at the global scale to share knowledge, data, tools, services, and to coordinate actions to ensure the interoperability of the resource system deployed at the local scale.

The $C P R$ is the model chosen to hold and govern the resource system. The participants (individuals or organisations with their own rules) must accept the rules to join the resource system and must contribute the required resources to do it, but they keep the ownership of the data, services, certifications, and tools they have contributed and the right to withdraw.

Nonetheless, as any other CPR, the eReuse.org commons is fragile. More precisely, it is congestion prone because the pool of reusable devices is subtractable and subject to free riding and abuse of the resource system due to competition among different stakeholders because eReuse.org is intentionally non-excludable. Thus, efficient and effective governance tools are needed to protect the core resource from depletion or congestion. The implementation is presented in Section 5 .

Analysing the design of long-enduring CPR institutions, Ostrom [18] identified eight principles, which are prerequisites for a sustainable CPR. We now discuss their application to our case.

1. Clearly defined boundaries. The fundamental principles of open and non-discriminatory access and open participation in the life of the community translate into prescriptions defined by the community licence and the specific collaboration agreements with all stakeholders, implemented by coordination software tools (applications and services) in the eReuse.org web platform. Governance tools should also regulate procedures for conflict resolution and economic compensation among all participants, which may be required to preserve the fundamental principles. These boundaries should be respected by the rules of each participating reuse community.

2. Rules regarding the appropriation and provision of common resources that are adapted to local conditions. The congruence between appropriation (usage of the resources: data and devices) and provision (contribution of information about devices) is mediated by the federation of asset management and allocation tools in each local community that develops an eReuse federated portal that assists in following up on devices across its circular life cycle. These tools embody and implement the principles defined by the eReuse community licence. Local reuse communities can have their own rules, procedures, and policies as long as they are compatible with the common rules of the federation (e.g., charges and policies for provision and appropriation of devices). Diversity in this case is an added value for adaptation to local conditions.

3. Collective-choice arrangements that allow most resource appropriators to participate in the decision- making process. Complexity and transaction costs grow as the network grows (number of devices, communities, and participants). This complexity should be managed by social structures that focus on each local community and on a global organisation. Beyond participation in local reuse communities, the governance of the federation naturally leads to a representative structure with a smaller executive board, a council with all stakeholders represented, and an advisory board with a wide representation of all views.

4. Effective monitoring by monitors who are part of, or accountable to, the appropriators. Monitoring is performed with the assistance of software tools that provide a common information base about the history and status of the common inventory of devices and with the lead of local trusted senior members that rely on open data to generate local and global indicators that are reported to appropriators. Monitoring applies to the federation, which means monitoring the contribution and usage of aggregated data and the operation of participants regarding the compliance of the community licence, while respecting the diversity of local conditions. These monitoring activities could result in audit certificates that increase trust in the federation from third parties.

5. Graduated sanctions for appropriators who do not respect community rules. The conflict-resolution system, not yet implemented, should have clear and systematic methods to deal with users that negatively affect the common resource system. Problems would typically arise among diverse stakeholders competing for the supply or the use of a set of devices or among participating organisations that are not contributing data about the devices they manage or use. These sanctions are planned to affect a reputation score given to each participant.

6. Conflict-resolution mechanisms that are cheap and easy to access. The conflict-resolution system should provide a structured procedure with several stages of escalation and progressive levels of complexity and economic cost to the parties, all driven by an expert (e.g., a lawyer or senior members) selected from a set of volunteers. Experience from other digital commons indicates this scheme can be cheap, easily accessible, efficient, effective, and scalable in addressing conflicts.

7. Self-determination of the community recognised by higher-level authorities. We plan the community licence to be examined by lawyers and written to be valid and enforceable under the local legislations where local eReuse member communities are deployed. That probably requires the establishment of local and global legal entities representing the community, probably in the legal form of a foundation.

8. In the case of larger CPRs, organisation in the form of multiple layers of nested enterprises, with small local CPRs at each base. There are plans to establish a legal entity, probably a foundation, to mediate among local members (such as individuals, professionals, and public entities), the many local CPR communities at the base, providing a federated CPR (second layer organisation) with many aspects in common that can interact with external organisations in the local and global scope.

\section{COMPUTING AS A SERVICE}

One key contribution from eReuse.org commons is the potential for a change of focus away from the property of de- 
vices to just using their services and to its standardisation. While, in some cases, owning a specific type of computer may be linked to social status, preference, or emotional attachment to a brand or model, many organisations have a functional view of computing, and computers are a standardised commodity that is counted in the number of work stations according to functional categories. Additionally, there is the long existing concept of distributed computing with lightweight client or terminal devices combined with network servers. In most cases, computer units become commodity boxes that can be easily replaced.

This particular context defines a business model where computer devices are reused and replaced to ensure the continuous operation of a number of workplaces. Organisations can adopt a simpler renting model where a payment is made for the temporary use of replaceable (not necessarily new) devices of specific characteristics, maintained and replaced by a third party, and therefore representing a fixed periodic cost. Cost sharing (collective purchase) schemes may allow reducing the initial cost of acquisition of new devices for the first user, even including any gain from the final recovery of materials. Incentives to maintain devices in good condition and promote other good practices, such as reporting transfers or final recycling, can be implemented through reputation scores or economic deposits that are returned at the end of each cycle of use. This model can help organisations achieve their environmental sustainability goals at much lower economic and environmental costs.

\section{IMPLEMENTATION}

The eReuse.org architecture defines a distributed system, instantiated by multiple organisations and implemented as software services, tools, and data repositories (Figure 3). It has the main objectives of 1) managing reuse and recycling actions, 2) ensuring the reuse and recycle processes by contributing to traceability, 3) facilitating third parties to join local eReuse communities (portals) by using, extending, and integrating the system, 4) generating inputs for indicators that measure circularity, and 5) maximising the performance of devices and their usage time. The architecture follows the governance principles presented in Section 3.

Device Diagnostic and Inventory: A set of tools to support the process of preparing for reuse and the inventory of digital devices. Preparation for reuse essentially consists of visual inspection, product safety (insuring voltage and other sector safety requirements), functionality tests (units must meet the ordinary use for which the item was originally placed), data deletion, generating meta data about traceability, and providing signed documents.

These tools include Hardware Discover, which obtains information from the computer and its components and generates a unique Hardware ID (HID) for each component and for the computer itself. The HID is a universally unique identifier, generated as a sequence of the serial number of a component and the manufacturer and model names. Eraser securely erases hard-drives and generates certificates. Benchmark performs quality tests to guarantee the capacity of the machine to correctly operate in a given workplace, including performance tests for the CPU or hard drive. Diagnosis performs diverse tests like 1) testing for failed sectors in the hard drive, estimating remaining life time, 2) testing RAM, hard-drive speeds, graphic capabilities, and CPU power with a real operating system. PXE server DeviceInventory is of- fered with a companion virtual image of a server that can be used simultaneously to massively register many computers connected to an Ethernet switch.

After executing all the desired tools, the Device Diagnostic and Inventory toolset generates a signed non-modifiable report with the results of the preparation for reuse process, ensuring that the process is kept totally automatic, and data cannot be modified by error or a malicious user. If the previous requirements are met, a certificate is issued and the item can be considered reusable (REEE). This report can be uploaded to a compatible IT asset management system (ITAMS) like DeviceHub.

DeviceHub: An ITAMS is focused on efficiently managing the circular life cycle of devices. It allows organisations to manage their hardware more effectively, avoiding unnecessary asset purchases (reduce), promoting the harvesting of existing resources (internal reuse), and interacting with external systems to manage the different processes involved in reusing and recycling, like disposal, external reusing, tracking, etc. These systems can be other ITAMS (like other DeviceHubs) or other solutions and traceability systems (like the global record of devices (GRD) global traceability database of eReuse.org). DeviceHubs are federated and autonomous. After the device is registered in a DeviceHub, the preparer or giver tags the computer by printing its identifier and a $\mathrm{QR}$ code that points to the uniform resource locator (URL) where the device description is stored. An example of instantiation of a DeviceHub is the Devicetag.io service (see Figure 4).

eReuse.org App: The eReuse.org app is a smartphone and tablet client for DeviceHub. It performs certain actions, like geolocating or visualising devices, that benefit from portability, camera, and geolocation features of smartphones and tablets and from registering them to a DeviceHub using a subset of Device Diagnostic and Inventory functionality. The main features are 1) registering new geolocated places into DeviceHub and assigning devices to them, 2) shortcuts to actions that change the state of devices (e.g., receive, recycle, and locate), and 3) obtain information about a device by scanning a printed QR code. The code is the URL of the device in the DeviceHub where it has been registered. Some of these actions require geolocation data, as they register where actions have been performed (Figure 5).

With DeviceHub and its client app, we can control different aspects that affect the reuse and recycling process. It uses an ID system that uses QR codes for identification and tracking purposes. After the device is registered, the preparer or giver tags the computer by printing its identifier and QR code that points to the URL where the device's description is stored. Then, with the eReuse.org app, a smartphone can be used to geolocate and visualise the information of any device.

Assurance of global traceability of devices is performed using two databases: the directory of collection points (DCP) and the global record of devices (GRD).

Directory of Collection Points (DCP): To guarantee final recycling of digital devices, the eReuse.org community geolocates all authorised collection points (see Figure 6) and asks their users to perform a geolocation action once they send devices to collection for recycling. If the last recorded geolocation has been done within the geographical area of known collection points, we can say with some certainty that the devices have been collected to be recycled. When that 


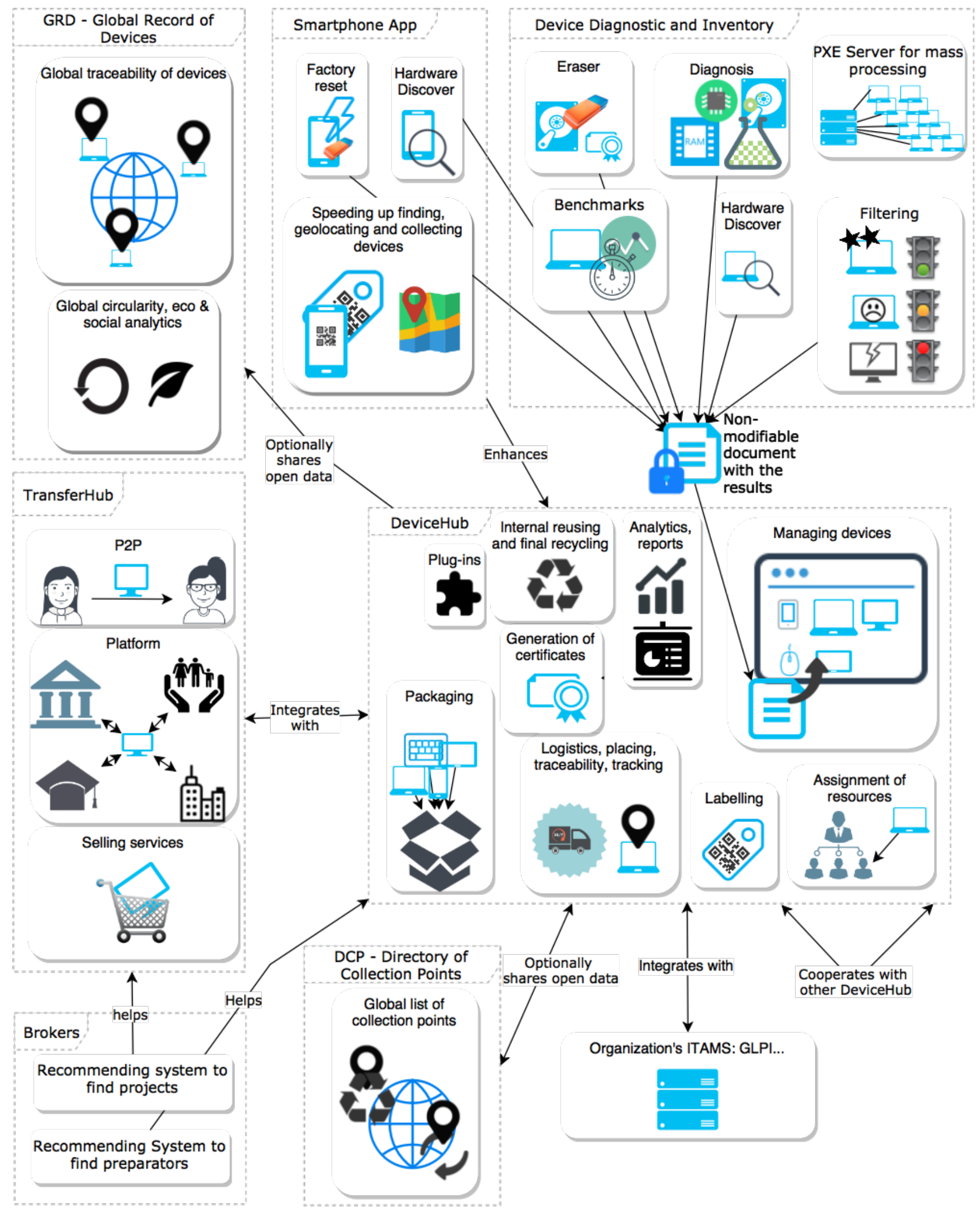

Figure 3: eReuse.org software components. 


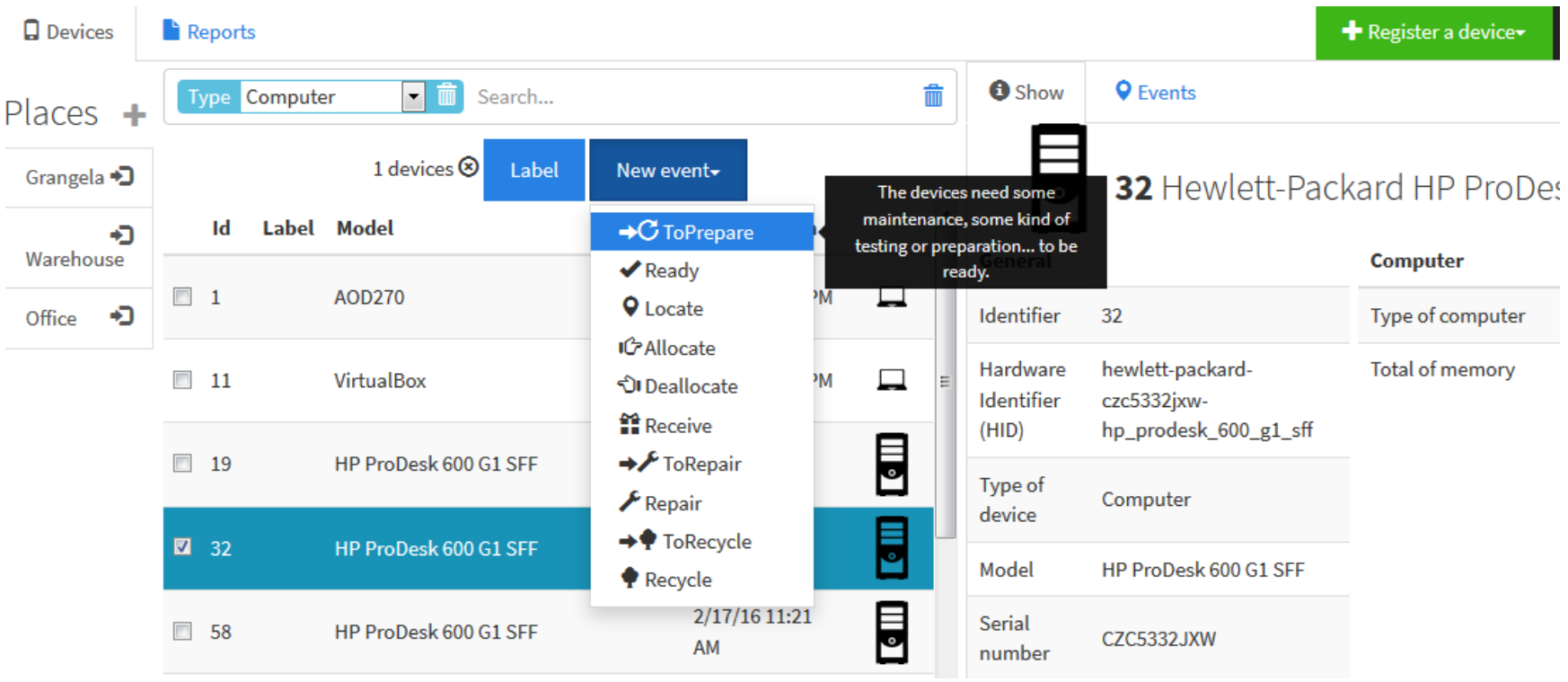

Figure 4: DeviceHub Client front-end.

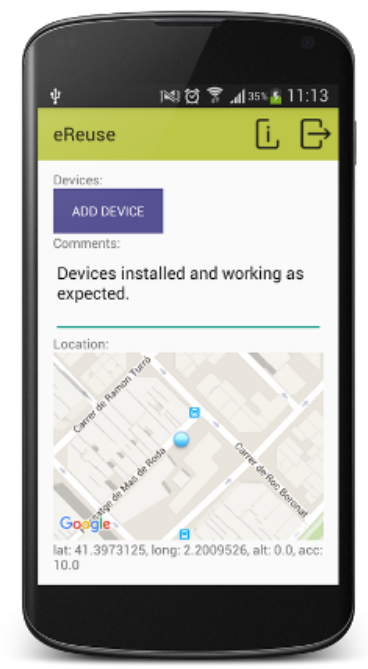

Figure 5: eReuse.org Android application.

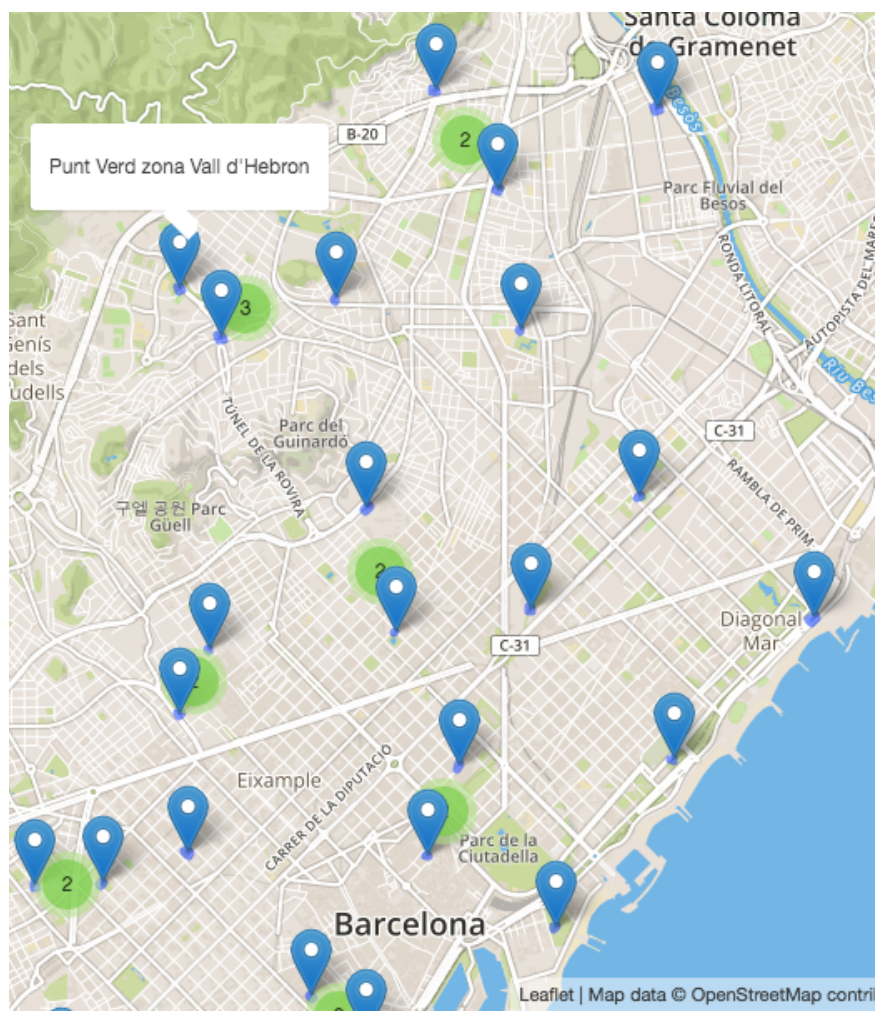

Figure 6: Recorded recycle collection points of Barcelona with DCP. 
action is performed by registered employees of a collection point, the certainty will be higher.

Global Record of Devices (GRD): An online log that maintains a global list of traceability information about devices, so it can assist if a device or component is lost or if there are similar problems. The GRD works with open data and is designed to be used with external users who want to report traceability information. At the same time, it aggregates traceability data with the goal to input the data for measuring circularity indicators. The GRD provides a REST API to allow ITAMS, as DeviceHub, to report about the life cycle of devices, environmental responsibility for organisations, etc. From each device, it collects the approximate geographical path it has followed (not in detail to preserve privacy), the DeviceHub where it has been stored (URL), the collection points where it was located before its final recycling, and the list of components in the case of computers.

TransferHub: TransferHub is a system that manages the distribution channel, focused on simplifying matchmaking and transferals in the reuse process. It announces receivers and givers, so they can find each other easily. Optionally, it can add regulation or transaction mechanisms. TransferHub does not manage the donation itself; it interacts with DeviceHub or similar ITAMS to acknowledge transfers and to retrieve the available devices. TransferHub can support three main transfer schemes:

1. Peer-to-peer, which enables transfers across individuals without any regulation scheme.

2. Social platform, which supports donation of devices to excluded collectives and to projects for social change. A social platform is an umbrella that federates specific DeviceHubs from donors, receivers, and other platforms, building an electronic reuse ecosystem. The main features are capturing offer (donations) and demand (social projects) to trade with reuse professional service providers (preparation, installation, and maintenance), a donation service (legal advice), a payment system, a social support crowdfunding, and a recommendation or reputation system.

3. Commerce, which adds the necessary mechanisms to get paid by the transferal, such as an online store.

Reutilitza.cat is a working prototype of a TransferHub with social platform functionality. In Catalonia, there are several successful experiences of public organisations, private companies, reuse centres, and social recipients exchanging services and goods. This platform is in close cooperation with the public waste agency, social enterprises that repair and refurbish equipment, and consumer groups guaranteeing the final recycling of the devices. The main tasks are finding donors willing to donate at no cost to social receivers, finding volunteers or professionals willing to prepare computers for reuse at donors' locations, disseminating social projects among donors, signing agreements between Reutilitza.cat and professionals, recording legal asset transfers to donors and receivers, ensuring financial sustainability of the platform with market prices for the services offered, and making agreements with other agents that can finance the platform, such as manufacturers (obligation to reuse) and public services (support social initiatives). Reutilitza.cat has 711 registered users; it has facilitated the donation of 1,640 digital devices to 909 social initiatives and registered so far more than 2,270 devices for eventual reuse (April 2016).

\subsection{A Resource System for Circular Economy}

Researchers, citizens, companies, and governments are building a resource system: a set of software tools, services, and open data around the life cycle of digital devices. To facilitate the understanding of the value generated, the process of data analysis is as follows. The Device Diagnostic and Inventory tool obtains information about a device, which is uploaded to a DeviceHub. Different DeviceHubs exchange traceability data about the devices they manage with GRD and DCP. Additionally, DCP provides knowledge regarding the locations for recycling, while GRD provides information about traceability and circularity information regarding devices, like durability of certain models, total usage time of devices, or the path they have followed after acquiring them until they have been recycled. This builds confidence in donors, governments, manufacturers, and donor organisations and is in line with recent European directives. Most importantly, it generates data that enables a transition towards a fully circular economy by building the knowledge base for environmental action and sustainability.

The information in the eReuse.org GRD lifecycle repository should allow deducting the number of times a device has been reused, its durability or elapsed time between first and last usage, the total time the device has been operating, and information regarding traceability. Apart from executing Device Diagnostic and Inventory on a computer, some of this information can be provided automatically to users by platforms using DeviceHub through programs installed on their devices. To preserve the privacy of users, eReuse.org only collects aggregated information in large periods of time, like the number of months a device has been used. This information can be used by the platform to rate the reputation of its receivers.

A person that finds any piece of electronic waste that has been monitored by eReuse.org can notify eReuse.org about it by sending its location. Leaked devices may not be in good condition. Therefore, there are many ways to identify them, including introducing the serial numbers manually, executing the Device Diagnostic and Inventory (a tool from eReuse.org), or scanning a printed QR code (placed on the device in the preparation for reuse process) with the eReuse.org smartphone app and automatically sending the GPS coordinates. While eReuse.org does not have information about the identification of the last owner, it knows the last ITAMS where the device was registered in, so it can be notified.

\section{CONCLUSIONS}

Digital devices, so widespread in our world, have become environmental risks as well as requirements to participate in society. Reuse is a key step to develop a circular ecology and economy that can extend the lifetime of these devices, ensuring a final proper recycling, while creating less costly devices for more people. This is a global issue, and the paper proposes a commons organisation and governance of a federation of communities working towards the circularity of digital devices, with a set of supporting software tools, services, and data sets that enable and optimise the effectiveness of these organisations.

We describe the mature, open-source, decentralised, local, 
scalable tools and services to optimise and certify preparation for reuse and to ensure traceability until recycling. Reuse centres have a set of support tools to facilitate the preparation and certification of devices for reuse (hardware rating, deletion of data, tests of operation, inventory, labelling, finding recipients, and packaging). Reusers have support tools to report further reuses and to finally recycle them at authorised points. Social enterprises and reuse and recycling centres can create their own instances (local web portals) or operate one as a cloud service. These should find sustainable models, offering donor services, such as preparation for reuse and maintenance services equivalent to an extended guarantee to social recipients or follow the computing as a service model. Our methods and the open data about durability allow providing better information to enable buyers to select products with high potential for reuse and to avoid components with too-short durability, while leaving the remaining for recycling.

We describe our current distributed ecosystem of federated and autonomous DeviceHub instances and support tools. A data exchange protocol is standardised to facilitate global traceability beyond locally known agents in the reuse chain and produce open data while preserving privacy. This allows third parties to use the aggregated open data in innovative ways, such as for research or potential audits. Citizens can analyse data concerning hardware tests and device and component durability and report on electronic waste landfills.

Future work will bring more experience in creating local initiatives, new business models, and more data and improvements to every aspect of the current model. We believe this ecosystem of tools, services, and data, organised as a commons, will help to mature the circular ecology of digital devices that our world desperately needs.

\section{ACKNOWLEDGMENTS}

This work is supported by the European Community Framework Programme 7, Collective Awareness Platforms for Sustainability and Social Innovation (CAPS), project "Collective enHanced Environment for Social Tasks" (CHEST), contract 611333 .

\section{REFERENCES}

[1] Apple. iPhone 5s Environmental Report, 2013.

[2] C. P. Balde, F. Wang, R. Kuehr, and J. Huisman. The global e-waste monitor - 2014. United Nations University, IAS - SCYCLE, Bonn, Germany, 2015.

[3] A. Cerrillo. Residus electrònics fora de control. La Vanguardia., 29.05:28-29, 2014.

[4] CWIT partners. Countering WEEE Illegal Trade Summary Report. CWIT project, Lyon, France, 2015.

[5] Department of Economic and Social Affairs of the United Nations Secretariat. Trends in Sustainable Development - Chemicals, Mining, Transport, Waste Management. United Nations, 2010.

[6] J. B. (Editor). Beacons of the information society: The alexandria proclamation on information literacy and lifelong learning. Technical report, UNESCO, in Alexandria, Egypt, 2005.

[7] E. M. Ercan. Global warming potential of a smartphone: Using life cycle assessment methodology. KTH Master of Science Thesis, 2013.
[8] Eurobarometer. Attitudes of europeans towards waste management and resource efficiency. In Flash Eurobarometer 388. European Commission, 2014.

[9] D. Franquesa, J. L. Cruz, C. Alvarez, F. Sanchez, A. Fernandez, and D. Lopez. The social and environmental impact of engineering solutions: from the lab to the real world. Int $J$ Eng Educ, 5(26):1144-1155, 2010.

[10] D. Franquesa, L. Navarro, D. López, X. Bustamante, and S. Lamora. Breaking barriers on reuse of digital devices ensuring final recycling. In EnviroInfo and ICT for Sustainability 2015. Atlantis Press, 2015.

[11] M. Güvendik. From Smartphone to Futurephone: Assessing the Environmental Impacts of Different Circular Economy Scenarios of a Smartphone Using LCA. TUDelft Institutional Repository, Aug. 2014.

[12] L. Maestri and R. Wakkary. Understanding repair as a creative process of everyday design. In Proceedings of the 8th ACM Conference on Creativity and Cognition, pages 81-90, New York, NY, USA, 2011. ACM.

[13] M.J. Goedkoop, R. Heijungs, M. Huijbregts, A. De Schryver, J. Struijs, R. Van Zelm. ReCiPe 2008, A life cycle impact assessment method which comprises harmonised category indicators at the midpoint and the endpoint level. First edition Report I: Characterisation, 6 January 2009.

[14] Nokia. Eco profile Nokia Lumia 820, 2012.

[15] Nokia. Eco profile Nokia Asha 311, 2013.

[16] Nokia. Eco profile Nokia Lumia 1520, 2013.

[17] O. G. F. on Environment Focusing on Sustainable Materials Management. Materials case study 1: Critical metals and mobile devices. Working document, October 2010.

[18] E. Ostrom. Governing the commons: the evolution of institutions for collective action. Cambridge University Press, Nov. 1990.

[19] C. Periou. Waste: the challenges facing developing countries - world production of municipal solid waste, 2012-2025. Private Sector \& Development, 1(15):1-6, October 2012.

[20] C. Remy and E. Huang. Limits and sustainable interaction design: Obsolescence in a future of collapse and resource scarcity. First Monday, 20(8), 2015.

[21] M. Schuelp, C. Hagelueken, and R. Kuehr. Recycling from e-waste to resources. United Nations Environ Program United Nations University, 2009.

[22] K. Toyama. Preliminary thoughts on a taxonomy of value for sustainable computing. First Monday, 20(8), 2015 . 